\section{Hipertensão arterial referida e utilização de medicamentos de uso contínuo no Brasil: um estudo de base populacional}

\author{
Self-reported hypertension and non-adherence to \\ continuous-use medication in Brazil: a population- \\ based study
}

\section{Hipertensión y consumo continuo de medicamentos en Brasil: un estudio de base en la población}

\author{
${ }^{1}$ Faculdade de Medicina, \\ Universidade Federal de \\ Minas Gerais, Belo Horizonte, \\ Brasil. \\ 2 Escola de Nutrição, \\ Universidade Federal de Ouro \\ Preto, Ouro Preto, Brasil. \\ Correspondência \\ R. A. Ferreira \\ Faculdade de Medicina, \\ Universidade Federal de \\ Minas Gerais. \\ Av. Alfredo Balena 190, Belo \\ Horizonte, MG 30130-100, \\ Brasil. \\ reginaraferreira@yahoo.com.br
}

\begin{abstract}
The aim of this study was to estimate the prevalence of non-adherence to several continuoususe drugs by patients 30 to 79 years of age with self-reported hypertension, and associated factors, drawing on data from the Brazilian $\mathrm{Na}$ tional Sample Household Survey (PNAD-2008). Prevalence ratios $(P R)$ and respective 95\% confidence intervals (95\%CI) were obtained by Pois son regression. The proportion of individuals that failed to adhere to continuous-use medication was $17 \%$. Characteristics directly associated with non-adherence were: male gender, residence in the North, Northeast, or Central-West of Brazil, and smoking. Non-adherence decreased with age, per capita family income, number of chronic diseases, and medical consultation in the previous 12 months, and was inversely associated with 11 or more years of schooling ( $P R$ = 0.92; 95\%CI: 0.86-0.98), not working and not being unemployed, and physical activity. Adherence to antihypertensive medication is necessary and requires investment in primary care, improved access to health services, and measures to offset regional, social, and gender inequalities.
\end{abstract}

Hypertension; Drugs of Continuous Use; Therapeutics
Reginara Alves Ferreira 1 Sandhi Maria Barreto 1 Luana Giatti 2

\section{Resumo}

O objetivo do presente estudo foi estimar a prevalência e fatores associados à não utilização de algum medicamento de uso contínuo nos indivíduos de 30-79 anos que referiram hipertensão arterial na Pesquisa Nacional por Amostra de Domicílios (PNAD-2008), Brasil. Razões de prevalência $(R P)$ e respectivos intervalos de $95 \%$ de confiança (IC95\%) foram obtidos por meio de regressão de Poisson. A proporção de indivíduos que não utilizava medicamentos de uso contínuo foi de $17 \%$. Características positivamente associadas à não utilização foram: sexo masculino, residência nas regiões Norte, Nordeste, Centro-oeste e hábito de fumar. O não uso desses medicamentos diminuiu com a idade, renda domiciliar per capita, número de doenças crônicas e de consulta médica nos últimos 12 meses; foi menor entre os que tinham 11 anos e mais de estudo ( $R P=0,92$; IC95\%: 0,86-0,98), nos que não trabalhavam nem estavam desempregados e nos que não realizavam atividade física. A adesão à terapia medicamentosa no controle de hipertensão arterial é necessária e requer investimento na atenção primária, na melhoria no acesso aos serviços de saúde e no enfretamento das desigualdades regionais, sociais e de gênero.

Hipertensão; Medicamentos de Uso Contínuo; Terapêutica 


\section{Introdução}

A hipertensão arterial sistêmica (HAS) é o principal fator de risco para as doenças cerebrovasculares, doenças isquêmicas do coração 1 e para a carga global de doenças em homens e mulheres de todas as idades 2. É a principal causa de óbito prevenível no mundo, responsável por $13 \%$ das mortes 1. Em 2000, a prevalência mundial foi estimada em torno de $26 \%$, devendo passar para $29 \%$ em 2025, considerando apenas o aumento populacional e a composição etária. Esse percentual equivale a aproximadamente 1,56 bilhão de pessoas afetadas ${ }^{3}$. Na América Latina, a HAS afeta mais de um terço da população 1 . No Brasil, em 2008, cerca de $21 \%$ das pessoas com 20 anos e mais relataram diagnóstico de HAS 4 e, em 2011, esse percentual chegou a quase $23 \%$ entre individuos com 18 anos 5 .

O tratamento adequado da HAS é fundamental para a redução da morbidade e mortalidade por doenças cardiovasculares e consiste em mudanças no estilo de vida e uso contínuo de um ou mais tipos de medicamentos antihipertensivos 6 . Nos países desenvolvidos, a proporção de indivíduos com hipertensão que realiza tratamento medicamentoso vem sofrendo alterações ao longo dos anos: no Canadá, aumentou de 35\% em 1992 para quase $80 \%$ em 20097 e nos estados Unidos de 60\% entre 1992 e 2002 para cerca de $70 \%$ entre 2005 e 20088 . Entre 1986 e 2001, a proporção de indivíduos com hipertensão arterial que utilizava medicamentos nos países em desenvolvimento variou de 10 a $50 \% 9$.

No Brasil, estudos com a população adulta mostraram que a proporção de pessoas com diagnóstico que não utilizava anti-hipertensivos variou de $10 \%$, no Rio Grande do Sul, no ano 2000 10, a 53\%, em Tubarão, Santa Catarina, no ano de 2003 11. Entre idosos residentes em Bambuí, Minas Gerais, em 1997, esse percentual chegou a $18 \%$ daqueles com diagnóstico conhecido da doença 12 .

Estudos indicam que a não utilização de medicamentos anti-hipertensivos parece estar relacionada com características sociodemográficas, especialmente com o sexo masculino e as idades mais jovens, com os comportamentos relacionados à saúde, como tabagismo e sedentarismo, com as condições de saúde dos indivíduos e com utilização dos serviços de saúde 8,12,13,14. Porém, não identificamos estudo de base populacional representativo da população brasileira que tenha investigado as características dos indivíduos com diagnóstico referido de hipertensão arterial que não fazem uso de medicamentos para controle da doença.
A Pesquisa Nacional por Amostra de Domicílios realizada no ano de 2008 (PNAD 2008) contém informações sobre hipertensão arterial referida e uso contínuo de medicamentos. Entretanto, não há informação específica sobre o tipo ou finalidade do medicamento utilizado 15 . A hipertensão arterial referida é um indicador simples, que pode ser usado quando a determinação da prevalência da hipertensão pela aferição da pressão arterial e uso de medicamentos anti-hipertensivos não é viável. Tem sido utilizada em inquéritos como o National Health and Nutrition Examination Survey (NHANES) nos Estados Unidos e o VIGITEL (Vigilância de Fatores de Risco e proteção para Doenças Crônicas por Inquérito Telefônico) no Brasil 5,15. Estudos de validação da hipertensão referida realizados em população idosa e adulta no país mostram valores de sensibilidade de $72,1 \%$ a $84,4 \%$ e especificidade de $86,4 \%$ a $87,5 \%$, respectivamente 16,17 .

O presente estudo tem como pressuposto que os indivíduos que relatam diagnóstico médico de hipertensão arterial deveriam utilizar medicamentos de uso contínuo, conforme orientam os protocolos de tratamento dessa condição 6 . Nesse sentido, a não utilização de medicamentos de uso contínuo é um marcador de não adesão ao tratamento medicamentoso da hipertensão.

Os objetivos deste estudo foram estimar a prevalência e os fatores associados à não utilização de medicamentos de uso contínuo entre indivíduos que relataram diagnóstico de hipertensão na PNAD 2008. Tem como principal hipótese que a não utilização de medicamentos de uso contínuo é mais frequente entre pessoas que relataram hipertensão arterial do sexo masculino, mais jovens, menos escolarizados, fumantes, que não realizam atividade física, que utilizam os serviços de saúde com menor frequência e que não apresentam outras morbidades referidas. Investigará ainda se o cadastramento na Unidade de Saúde da Família está associado a uma frequência maior de uso de medicamento contínuo.

\section{Métodos}

\section{População do estudo}

A população do estudo é composta por indivíduos de 30-79 anos que participaram da PNAD realizada no ano de 2008 pelo Instituto Brasileiro de Geografia e Estatística (IBGE) e que referiram ter hipertensão ao responderem "sim" à pergunta "algum médico ou profissional da saúde disse que ... tem hipertensão (pressão alta)?”. Indivíduos com idade inferior a 30 anos foram excluídos 
devido à menor prevalência de HAS (inferior a $4 \%$ nesta faixa etária) 18 e à maior probabilidade de ausência de diagnóstico. Já aqueles com 80 anos e mais foram excluídos pela maior frequência de comorbidades que levam ao uso de medicamento.

A PNAD obtém anualmente informações sobre diversos aspectos da população brasileira, incluindo características socioeconômicas, trabalho, migração, dentre outros. Em 2008, foram obtidas informações sobre condições de saúde que incluíram morbidade referida, utilização de serviços de saúde e alguns comportamentos relacionados à saúde. A pesquisa tem múltiplos propósitos e adota um plano amostral estratificado e conglomerado com probabilidades desiguais de seleção em um, dois ou três estágios de seleção, dependendo do estrato. Obtém-se uma amostra probabilística de domicílios representativa da população brasileira em que todos os moradores são investigados por meio de entrevista. Informantes secundários, pessoas com 14 anos ou mais que se encontravam no domicílio no momento da entrevista, forneceram informações sobre os moradores ausentes ${ }^{15}$. Para informações mais detalhadas verificar em Silva et al. 19 e IBGE 15. Em 2008, foram entrevistadas 391.868, sendo 183.452 adultos com idade de 30-79 anos e 47.268 (26,2\%) referiram ter hipertensão. Eles constituem a população deste estudo.

\section{Variáveis}

A variável resposta deste estudo foi a utilização de medicamentos de uso contínuo (sim ou não) obtida por meio da pergunta "utiliza medicamentos de uso contínuo?".

Três conjuntos de variáveis explicativas foram incluídas:

1) Características sociodemográficas: sexo (masculino e feminino), idade em faixas etárias (30-39, 40-49, 50-59, 60-69,70-79), escolaridade em anos completos de estudo (0-3, 4-7, 8-10, 11 e mais), região de residência (urbano ou rural), macrorregião de residência (Sudeste, Sul, Centro-oeste, Nordeste e Norte), trabalho na semana de referência - 21 a 27 de setembro de 2008 (trabalha, desempregado e não trabalha nem está desempregado), renda domiciliar per capita em reais, agrupada em quintis. Considerando o pequeno percentual de indivíduos que relatou cor/raça amarela $(0,6 \%)$ e indígena $(0,4 \%)$, optou-se por agrupar as categorias da seguinte forma: branco / amarelo, preto e pardo/indígena;

2) Comportamentos relacionados à saúde: tabagismo atual, considerando-se "fumante" o indivíduo que fumou pelo menos 100 cigarros durante toda a vida e fuma atualmente, "ex-fumante" o indivíduo que fumou pelo menos 100 cigarros durante toda a vida, mas não fuma atualmente e "não fumante" aquele que fumou menos de 100 cigarros durante toda a vida ou nunca fumou; prática de atividade física regular (não, sim), considerando-se entre os que não praticam aqueles indivíduos que não realizavam qualquer atividade física ou realizavam alguma atividade até duas vezes por semana, independentemente da frequência e da intensidade;

3) Saúde: presença de doenças crônicas que corresponde ao relato de outras morbidades (diabetes, doença do coração, doença renal crônica, depressão) categorizada em: "somente hipertensão", "hipertensão mais uma doença crônica”, "hipertensão mais duas doenças crônicas"; número de consultas médicas nos últimos 12 meses (nenhuma, 1, 2-3, 4 e mais), relato de internações hospitalares nos últimos 12 meses (sim, não), e o cadastro nas Unidades de Saúde da Família (sim, não). Plano de saúde foi considerada uma variável intermediária na associação entre uso de medicamento contínuo e utilização de serviços de saúde, por isso não será incluída nesta análise.

\section{Análise}

Inicialmente, foi realizada análise descritiva das características sociodemográficas dos participantes do estudo e estimadas as prevalências de não utilização de medicamento de uso contínuo segundo as variáveis explicativas. A associação entre a não utilização de medicamento de uso contínuo e as variáveis explicativas foi medida por meio do teste do qui-quadrado de Pearson, com nível de 0,05 de significância.

A seguir, foi realizada análise univariada por meio da razão de prevalência e seu intervalo de 95\% de confiança (IC95\%) com uso da regressão de Poisson. A razão de prevalência foi utilizada por conta da prevalência do evento de interesse. Todos os fatores associados à não utilização de medicamento de uso contínuo, com nível de significância de 0,20 na análise univariada, foram incluídos na análise multivariada por blocos de variáveis, permanecendo no modelo final as variáveis com o valor de $\mathrm{p}<0,05$. A análise dos dados foi realizada com uso de procedimentos para a análise de inquéritos populacionais do programa Stata 10 (Stata Corp., College Station, Estados Unidos), que incorporam os pesos distintos das observações, corrigem diferentes probabilidades de seleção e produzem estimativas mais corretas do erro padrão, uma vez que ele é influenciado pela conglomeração das unidades derivadas do delineamento amostral de múltiplos estágios da PNAD 19. 


\section{Resultados}

\section{Características gerais da população}

Entre os indivíduos com idade de 30-79 anos que referiram ter hipertensão na PNAD 2008, a média da idade foi de 55,7 anos (IC95\%: 55,6-55,8). Aproximadamente $60 \%$ dos indivíduos eram do sexo feminino, $50 \%$ relataram cor/raça branca/ amarela, $65 \%$ estudaram no máximo sete anos, $86 \%$ residiam em centros urbanos e cerca de $48 \%$ não trabalhavam e nem estavam desempregados. Em torno de $16 \%$ eram fumantes, mais de $85 \%$ não praticavam atividade física e $4 \%$ apresentavam três ou mais doenças crônicas. Quase $50 \%$ dos indivíduos consultaram o médico mais de quatro vezes nos últimos 12 meses e $13 \%$ estiveram internados nesse período. Metade da população estava registrada em Unidades de Saúde da Família (Tabela 1).
Prevalência e fatores associados ao não uso de medicamentos

A proporção de indivíduos que relatou não fazer uso medicamento de uso contínuo foi de $17 \%$. A Tabela 1 apresenta as porcentagens de não utilização de medicamentos de uso contínuo de acordo com as características sociodemográficas, comportamentos relacionados à saúde, condições de saúde e uso dos serviços de saúde para a população total do estudo. Apenas a variável registro na unidade de saúde da família não foi estatisticamente associada à não utilização de medicamentos.

À análise univariada, a frequência de indivíduos que não utilizavam medicamentos de uso contínuo diminuiu com a idade foi maior entre os homens, os que relataram de 8 a 10 anos de estudo, os que referiram cor da pele/raça preta e os pardos/indígenas, os que viviam nas regiões

Tabela 1

Prevalência e fatores associados à ausência do uso de medicamento contínuo, segundo características sociodemográficas, comportamentos relacionados à saúde e situação de saúde entre indivíduos de 30-79 anos que referiram hipertensão arterial sistêmica. Pesquisa Nacional por Amostra de Domicílios, 2008, Brasil.

\begin{tabular}{|c|c|c|c|c|}
\hline Variável & $\begin{array}{l}\text { População } \\
\text { n (\%) }\end{array}$ & $\begin{array}{l}\text { Não utilização de medicamento } \\
\text { de uso contínuo (\%) }\end{array}$ & Valor de p & RP (IC95\%) \\
\hline \multicolumn{5}{|l|}{ Características sociodemográficas } \\
\hline \multicolumn{5}{|l|}{ Sexo } \\
\hline Masculino & $18.649(39,7)$ & 22,5 & & 1,00 \\
\hline Feminino & $28.619(60,3)$ & 13,4 & $<0,0001$ & $0,59(0,57-0,62)$ \\
\hline \multicolumn{5}{|l|}{ Idade (em anos) } \\
\hline $30-39$ & $5.153(10,6)$ & 42,3 & & 1,00 \\
\hline $40-49$ & $9.974(20,7)$ & 24,7 & & $0,58(0,55-0,61)$ \\
\hline $50-59$ & $13.285(28,1)$ & 14,5 & & $0,34(0,32-0,36)$ \\
\hline $60-69$ & $11.570(24,9)$ & 9,1 & & $0,22(0,20-0,23)$ \\
\hline $70-79$ & $7.286(15,8)$ & 6,8 & $<0,0001$ & $0,16(0,14-0,18)$ \\
\hline \multicolumn{5}{|l|}{ Cor/Raça } \\
\hline Branca/Amarela & $22.504(51,2)$ & 14 & & 1,00 \\
\hline Preta & $4.638(9,3)$ & 18,8 & & $1,34(1,25-1,44)$ \\
\hline Parda/Indígena & $20.101(39,5)$ & 20,4 & & $1,45(1,39-1,52)$ \\
\hline Sem informação & 25 & & $<0,0001$ & \\
\hline \multicolumn{5}{|l|}{ Escolaridade (anos de estudo) } \\
\hline Nenhuma ou até 3 & $17.548(37,4)$ & 16,2 & & 1,00 \\
\hline $4-7$ & $12.937(28,3)$ & 16,8 & & $1,04(0,99-1,10)$ \\
\hline $8-10$ & $5.658(11,7)$ & 20,2 & & $1,25(1,17-1,34)$ \\
\hline 11 e mais & $11.007(22,7)$ & 16,9 & $<0,0001$ & $1,04(0,99-1,11)$ \\
\hline Sem informação & 118 & & & \\
\hline \multicolumn{5}{|l|}{ Trabalho na semana de referência } \\
\hline Trabalhou & $24.180(50,8)$ & 23,2 & & 1,00 \\
\hline Desempregado & $995(2,0)$ & 27,9 & & $1,21(1,08-1,40)$ \\
\hline Não trabalhou nem estava desempregado & $22.093(47,2)$ & 9,9 & $<0,0001$ & $0,43(0,41-0,45)$ \\
\hline
\end{tabular}

(continua) 
Tabela 1 (continuação)

\begin{tabular}{|c|c|c|c|c|}
\hline Variável & $\begin{array}{l}\text { População } \\
\text { n (\%) }\end{array}$ & $\begin{array}{c}\text { Não utilização de medicamento } \\
\text { de uso contínuo (\%) }\end{array}$ & Valor de $p$ & RP (IC95\%) \\
\hline \multicolumn{5}{|c|}{ Características sociodemográficas } \\
\hline \multicolumn{5}{|c|}{ Renda domiciliar per capita (em quintis) } \\
\hline 1ㅇ (menor quintil) & $9.457(19,4)$ & 26,5 & & 1,00 \\
\hline 2o & $8.869(18,9)$ & 19,8 & & $0,75(0,70-0,79$ \\
\hline 3으 & $8.988(20,0)$ & 14,7 & & $0,56(0,52-0,59$ \\
\hline 4으 & $9.100(20,9)$ & 14,8 & & $0,56(0,52-0,60$ \\
\hline 5o (maior quintil) & $9.097(20,7)$ & 10,4 & $<0,0001$ & $0,39(0,36-0,42$ \\
\hline Sem informação & 1.757 & & & \\
\hline \multicolumn{5}{|l|}{ Região de residência } \\
\hline Urbana & $40.648(85,6)$ & 16,4 & & 1,00 \\
\hline Rural & $6.620(14,4)$ & 20,6 & $<0,0001$ & $1,26(1,19-1,33$ \\
\hline \multicolumn{5}{|l|}{ Macrorregião de residência } \\
\hline Sudeste & $16.544(48,4)$ & 13,5 & & 1,00 \\
\hline Sul & $7.839(15,9)$ & 13,6 & & $1,00(0,94-1,08$ \\
\hline Centro-oeste & $4.980(6,7)$ & 19,1 & & $1,41(1,32-1,52$ \\
\hline Nordeste & $13.738(23,9)$ & 29,5 & & $2,19(2,05-2,34$ \\
\hline Norte & $4.167(5,1)$ & 23,1 & $<0,0001$ & $1,17(1,62-1,80$ \\
\hline \multicolumn{5}{|c|}{ Comportamentos relacionados à saúde } \\
\hline \multicolumn{5}{|c|}{ Tabagismo atual } \\
\hline Não fuma & $26.711(56,6)$ & 16 & & 1,00 \\
\hline Ex-fumante & $12.868(26,9)$ & 13,9 & & $0,87(0,82-0,92$ \\
\hline Fuma & $7.524(16,4)$ & 25,3 & $<0,0001$ & $1,58(1,50-1,66$ \\
\hline Sem informação & 165 & & & \\
\hline \multicolumn{5}{|l|}{ Prática de atividade física } \\
\hline Não & $40.491(86,2)$ & 18 & & 1,00 \\
\hline $\operatorname{Sim}$ & $6.777(13,8)$ & 10,8 & $<0,0001$ & $0,60(0,56-0,65$ \\
\hline \multicolumn{5}{|c|}{ Condições de saúde e utilização dos serviços } \\
\hline \multicolumn{5}{|c|}{ de saúde } \\
\hline \multicolumn{5}{|l|}{ Presença de doenças crônicas } \\
\hline Somente hipertensão & $33.708(70,8)$ & 20,4 & & 1,00 \\
\hline Hipertensão e mais uma & $11.546(24,8)$ & 9,2 & & $0,44(0,42-0,48$ \\
\hline Hipertensão e mais duas & $2.014(4,4)$ & 5,7 & $<0,0001$ & $0,28(0,23-0,34$ \\
\hline \multicolumn{5}{|c|}{ Número de consultas médicas nos últimos } \\
\hline \multicolumn{5}{|c|}{12 meses } \\
\hline 0 & $5.939(12,3)$ & 40,8 & & 1,00 \\
\hline 1 & $5.415(11,5)$ & 25,8 & & $0,63(0,60-067)$ \\
\hline $2-3$ & $13.482(28,7)$ & 17,4 & & $0,43(0,40-0,45$ \\
\hline 4 e mais & $22.432(47,5)$ & 8,4 & $<0,0001$ & $0,21(0,20-0,22$ \\
\hline \multicolumn{5}{|c|}{ Ocorrência de internações nos últimos 12} \\
\hline \multicolumn{5}{|c|}{ meses } \\
\hline Não & $41.328(87,3)$ & 17,9 & & 1,00 \\
\hline $\operatorname{Sim}$ & $5.940(12,7)$ & 10,7 & $<0,0001$ & $0,60(0,55-0,65$ \\
\hline \multicolumn{5}{|c|}{ Registro na unidade de saúde da família } \\
\hline Não & $22.934(49,5)$ & 17 & & 1,00 \\
\hline Sim & $24.257(50,5)$ & 17 & 0,908 & $1,00(0,96-1,05$ \\
\hline
\end{tabular}

IC95\%: intervalo de 95\% de confiança; RP: razão de prevalência. 
rurais e nas macrorregiões Centro-oeste, Nordeste e Norte do Brasil. Entre os desempregados, a frequência dos que não utilizavam medicamentos foi $20 \%$ superior do que entre os que trabalhavam. Já entre os que não trabalhavam nem estavam desempregados, tal frequência foi cerca de 57\% menor. Quanto menor a renda, maior a frequência de indivíduos que não faziam uso de medicamentos de uso contínuo (Tabela 1).

A não utilização de medicamentos de uso contínuo foi mais frequente entre os fumantes e menor entre os ex-fumantes quando comparados aos não fumantes. Também foi mais frequente entre os indivíduos que não realizavam atividade física quando comparados àqueles que praticavam (Tabela 1).

Em relação às condições de saúde, a não utilização de medicamentos de uso contínuo diminuiu mediante o aumento do número de doenças crônicas e do número de consultas médicas nos últimos 12 meses e foi menor entre os indivíduos que relataram internação nos últimos 12 meses (Tabela 1).

À análise multivariada realizada por blocos de variáveis explicativas, a não utilização de medicamentos se manteve estatisticamente associada ao sexo masculino, diminuiu com o aumento da idade, da renda domiciliar per capi$t a$, foi menor entre os indivíduos que tinham 11 anos e mais de estudo, entre os que não trabalhavam e não estavam desempregados e maior entre os que residiam nas macrorregiões Norte, Nordeste e Centro-oeste. Os comportamentos relacionados à saúde estudados permaneceram estatisticamente associados à não utilização de medicamentos, assim como a presença de doenças crônicas e de consultas médicas realizadas nos últimos 12 meses (dados não apresentados).

$\mathrm{Na}$ análise multivariada final, que incluiu todas as variáveis estatisticamente significantes em cada um dos grupos analisados (Tabela 2), a não utilização de medicamentos de uso contínuo se manteve significativamente maior entre os homens, os que residiam nas macrorregiões Norte, Nordeste e Centro-oeste e entre os fumantes. A prevalência de não uso de medicamentos diminuiu com o aumento da idade, da renda domiciliar per capita, com o número de doenças crônicas e de consultas médicas realizadas nos últimos 12 meses e, foi menor entre os que tinham 11 anos ou mais de estudo, os que não trabalhavam nem estavam desempregados e os que realizavam atividade física.

\section{Discussão}

Os resultados do presente estudo apontaram que cerca de $17 \%$ dos indivíduos que referiram diagnóstico de hipertensão arterial não utilizavam medicamentos de uso contínuo. A prevalência de não uso de medicamentos foi maior entre os homens, nas pessoas residentes nas regiões Centro-oeste, Norte e Nordeste e entre os fumantes. Com o aumento da idade, observou-se redução significativa do não uso, assim como entre os indivíduos mais escolarizados e de acordo com a elevação da renda domiciliar per capita. Ainda, menores prevalências foram verificadas entre os que não trabalhavam nem estavam desempregados à época da pesquisa, nos ex-fumantes e nos que referiram prática de atividade física. $\mathrm{Na}$ presença de comorbidades, a prevalência de não uso de medicamentos foi significativamente menor e se encontrou redução da prevalência com o aumento do número de consultas médicas realizadas no ano prévio.

Quase um quinto dos adultos com hipertensão arterial referida estudados relatou não fazer uso contínuo de algum medicamento. É bem possível que essa prevalência esteja subestimada, pois os indivíduos que informaram utilizar medicamentos de uso contínuo podem fazê-lo por algum motivo de saúde não abordado neste estudo. Entre os indivíduos que declararam ter apenas HAS, o percentual foi de $20 \%$. Ainda assim, o valor pode estar subestimado, uma vez que a dificuldade de entendimento sobre o que vem a ser utilizar medicamento de uso contínuo pode levar a informações incorretas.

O percentual de não utilização de medicamentos encontrado neste estudo foi próximo ao verificado no Canadá, onde cerca de $20 \%$ dos indivíduos que tiveram diagnóstico de hipertensão não utilizavam anti-hipertensivos 7 e inferior ao observado nos Estados Unidos (em torno de 30\%) 8 e em outros países em desenvolvimento 1 . A prevalência aqui identificada foi similar aos valores revelados entre os idosos residentes em Bambuí, Minas Gerais, em 1997 (18\% de quem conhecia sua condição) 12. Entretanto, foi maior que a registrada entre os idosos aposentados moradores em Belo Horizonte (11\%) no ano de 200320 e em adultos residentes no Rio Grande do Sul em 2000 (10\%) 10. Ao serem comparadas as porcentagens de não uso de medicamentos entre esses estudos, deve-se ter em conta as diferenças relativas às faixas etárias, aos períodos em que foram realizados, às características regionais e locais que envolvem tanto a organização dos serviços de saúde quanto os aspectos culturais, às metodologias utilizadas para aferir a não utilização de medicamentos, além 
Tabela 2

Fatores associados à ausência do uso de medicamento contínuo entre os indivíduos com idade entre 39 e 79 anos que declararam diagnóstico de hipertensão arterial sistêmica. Pesquisa Nacional por Amostra de Domicílios, 2008, Brasil.

\begin{tabular}{|c|c|c|}
\hline Variável & RP & $\%(I C 95 \%)$ \\
\hline \multicolumn{3}{|l|}{ Sexo } \\
\hline Masculino & 1,00 & \\
\hline Feminino & 0,76 & $0,73-0,80$ \\
\hline \multicolumn{3}{|l|}{ Idade (anos) } \\
\hline 30-39 & 1,00 & \\
\hline $40-49$ & 0,65 & $0,62-0,69$ \\
\hline $50-59$ & 0,44 & $0,41-0,47$ \\
\hline $60-69$ & 0,31 & $0,29-0,34$ \\
\hline 70-79 & 0,25 & $0,23-0,28$ \\
\hline \multicolumn{3}{|l|}{ Escolaridade (anos de estudo) } \\
\hline Nenhuma ou até 3 & 1,00 & \\
\hline $4-7$ & 0,97 & $0,92-1,02$ \\
\hline $8-10$ & 1,00 & $0,94-1,07$ \\
\hline 11 e mais & 0,92 & $0,86-0,98$ \\
\hline \multicolumn{3}{|l|}{ Trabalho na semana de referência } \\
\hline Trabalha & 1,00 & \\
\hline Desempregado & 1,02 & $0,92-1,14$ \\
\hline Não trabalhou, nem estava desempregado & 0,78 & $0,74-0,83$ \\
\hline \multicolumn{3}{|l|}{ Renda domiciliar per capita (em quintis) } \\
\hline 1으 & 1,00 & \\
\hline 2oㅇ & 0,93 & $0,88-0,98$ \\
\hline 3으 & 0,86 & $0,80-0,91$ \\
\hline 4으 & 0,85 & $0,80-0,91$ \\
\hline 5으 & 0,65 & $0,60-0,71$ \\
\hline \multicolumn{3}{|l|}{ Macrorregião de residência } \\
\hline Sudeste & 1,00 & \\
\hline Sul & 1,00 & $0,93-1,06$ \\
\hline Centro-oeste & 1,2 & $1,13-1,29$ \\
\hline Nordeste & 1,42 & $1,35-1,49$ \\
\hline Norte & 1,78 & $1,67-1,89$ \\
\hline \multicolumn{3}{|l|}{ Tabagismo atual } \\
\hline Não fuma & 1,00 & \\
\hline Ex-fumante & 0,94 & $0,89-0,99$ \\
\hline Fumante & 1,22 & $1,17-1,29$ \\
\hline \multicolumn{3}{|l|}{ Prática de atividade física } \\
\hline Não & 1,00 & \\
\hline Sim & 0,75 & $0,70-0,81$ \\
\hline \multicolumn{3}{|l|}{ Número de doenças crônicas } \\
\hline Somente hipertensão & 1,00 & \\
\hline Hipertensão e mais 1 & 0,66 & $0,62-0,70$ \\
\hline Hipertensão e 2 ou mais & 0,49 & $0,40-0,59$ \\
\hline \multicolumn{3}{|l|}{ Número de consultas médicas nos últimos } \\
\hline \multicolumn{3}{|l|}{12 meses } \\
\hline 0 & 1,00 & \\
\hline 1 & 0,69 & $0,65-0,73$ \\
\hline $2-3$ & 0,52 & $0,50-0,55$ \\
\hline 4 e mais & 0,3 & $0,28-0,32$ \\
\hline
\end{tabular}

IC95\%: intervalo de 95\% de confiança; RP: razão de prevalência. 
das transformações ocorridas na estrutura do sistema de saúde brasileiro nas últimas décadas. Em particular, vale ressaltar que a prevalência estimada neste estudo, por se basear em indicador que subestima a não adesão ao tratamento medicamentoso da hipertensão, indica que a real prevalência de não adesão no país deve ser superior àquelas previstas com base nos estudos citados. Possivelmente, isso se explica pela inclusão no presente estudo de regiões do país com piores indicadores de saúde geral como o Norte e o Nordeste 21,22.

Diversos estudos mostram que a utilização de medicamentos anti-hipertensivos é menor entre os indivíduos do sexo masculino 8,12,14,23,24. Em nosso estudo, a menor porcentagem de não utilização de medicamentos entre as mulheres pode ser, em parte, atribuída à utilização de contraceptivos orais. Infelizmente, não há informação na PNAD que permita descartar essa possibilidade, porém essa hipótese influenciaria apenas as mulheres mais jovens e parece não ser muito relevante, visto que o uso contínuo de medicamento foi maior entre as mulheres mais velhas. A utilização de serviços de saúde no Brasil, inclusive serviços farmacêuticos, é superior entre as mulheres 25,26, que tendem a utilizar mais medicamentos que os homens, independentemente do uso de anticoncepcionais 27. Essa diferença pode ser explicada por aspectos culturais, biológicos e reprodutivos que levam as mulheres a se preocuparem mais com a própria saúde 28,29, pelo papel de cuidador atribuído a elas particularmente no que tange à saúde de filhos e familares 13 e por apresentarem maior conhecimento geral sobre saúde que os homens 14,23. Além disso, é importante apontar que a percepção de efeitos colaterias causados pelos medicamentos anti-hipertensivos, como disfunção sexual causada por algumas classes medicamentosas 30,31 pode ter maior impacto negativo na adesão masculina, acentuando as desigualdades de gênero no uso desses medicamentos.

A associação entre idade mais jovem e não utilização de medicamento, especificamente anti-hipertensivos. também foi observada em estudos realizados em outros países 8,14,32 e no Brasil 12. A presença e o número de doenças crônicas cresce com o avanço da idade 33 , resultando em maior procura pelos serviços de saúde 34 e aumentando a necessidade de adesão ao tratamento da HAS. Embora as associações observadas sejam independentes, verificou-se que a presença de comorbidade esteve associada ao maior uso de anti-hipertensivo como relatado em outros estudos 12,14.

Em relação às características socioeconômicas estudadas, encontrou-se que a renda do- miciliar per capita influenciou o uso de medicamentos de forma mais importante do que a escolaridade. Já foi descrito que menores níveis de escolaridade estão associados à menor utilização de medicamentos anti-hipertensivos 14 . No presente trabalho, somente escolaridade acima de 11 anos de estudo apresentou associação inversa com a não utilização de medicamentos, e ainda assim de pequena intensidade. A escolaridade influencia positivamante a saúde pelo maior acesso à informação, por meio da adoção de comportamentos saudáveis e do uso dos serviços de saúde 25,34 e pode facilitar a compreensão da necessidade da utilização do medicamento e da maneira correta de fazê-lo. Mas a renda parece influenciar mais fortemente o acesso aos serviços do que a escolaridade 22 e também o tratamento da HAS 12,23. Além disso, ressalta-se que o custo do medicamento é importante para a adesão ao tratamento da HAS 31 . Entre os idosos com hipertensão em Belo Horizonte, a falta de recurso financeiro foi a principal causa de não uso dos medicamentos necessários 20 .

A implantação do Sistema Único de Saúde (SUS) e as ações que vêm sendo adotadas como o crescimento da atenção básica e o acesso gratuito aos medicamentos 35 possivelmente impactaram positivamente na utilização de medicamento anti-hipertensivo. No entanto, é preciso ressaltar que persistem limitações na disponibilização de medicamentos. De acordo com os dados da PNAD 2008, houve prescrição de medicamentos em $72 \%$ dos atendimentos realizados no SUS nos 15 dias anteriores à entrevista. Desses, apenas $45 \%$ das pessoas obtiveram gratuitamente todos os medicamentos 36. É possível que a influência da renda domiciliar na utilização de medicamentos tenha diminuído nos últimos anos por causa da ampliação da lista de medicamentos disponibilizados gratuitamente pelo programa Aqui tem Farmácia Popular realizada no ano de 2011 37. Outros aspectos como o desconhecimento do direito ao medicamento e a falta de adequação da prescrição médica às condições financeiras e necessidades dos indivíduos também podem dificultar a utilização de medicamentos de uso contínuo.

O fato de o não uso de medicamentos ser menos frequente entre aqueles que não trabalhavam nem procuravam por trabalho pode em parte ser explicado por efeitos dos próprios antihipertensivos. O uso de medicamentos diuréticos, por exemplo, pode dificultar a rotina de trabalho. Além disso, pessoas que trabalham, sobretudo os inseridos no trabalho sem proteção social, ou que estão à procura de emprego usam menos os serviços de saúde que as que não estão trabalhando 22,38. A proporção de indivíduos que 
não consultou o médico nos últimos 12 meses neste estudo foi menor entre os trabalhadores e desempregados (dados não mostrados), o que, provavelmente influencia a obtenção e a utilização de medicamentos de uso contínuo. Em geral, o horário de funcionamento dos serviços de atenção primária é incompatível com o horário habitual de trabalho. podendo influenciar o uso dos serviços de saúde 29 . É possível que esses fatores associados a piores condições de saúde expliquem a maior utilização de serviços entre os que não trabalhavam e não estavam desempregados e o maior uso de medicamentos. Já os indivíduos que estão desempregados podem se encontrar desmotivados para o cuidado com a saúde. levando ao menor uso dos serviços $38 \mathrm{e}$ também de medicamentos.

Ao contrário de estudos realizados na China 24 e Costa Rica 14, não se verificou diferença na utilização de medicamentos de uso contínuo entre as regiões rurais e urbanas e sim entre as cinco macrorregiões do país. $\mathrm{O}$ acesso aos serviços de saúde no Brasil sofre grande influência do local onde as pessoas residem. A chance de os indivíduos residentes nas macrorregiões Norte, Nordeste e Centro-oeste que buscam atendimento não conseguirem obtê-lo é maior do que os que moram nas regiões Sul e Sudeste 21,22. Da mesma forma, o acesso aos medicamentos pode ser mais restrito nessas regiões do país, tanto em razão da indisponibilidade do medicamento quanto ao menor acesso à prescrição.

A associação inversa entre o número de consultas médicas e a não utilização de medicamentos de uso contínuo sugere a importância dos serviços e equipe de saúde na adesão ao tratamento. Tal associação foi encontrada em estudos sobre o uso de anti-hipertensivos 8,12, bem como para a utilização de medicamentos de uso contínuo ${ }^{28}$. O uso dos serviços de saúde além de ampliar o acesso aos medicamentos, promove, por meio da interação com os profissionais de saúde, maior conhecimento da doença e aceitação do tratamento, seja medicamentoso ou não. O conhecimento do indivíduo sobre a doença e o tratamento pode facilitar o início e a adesão ao tratamento de doenças crônicas, contribuindo para a utilização contínua de medicamentos 31 .

Apesar da importância do vínculo com a equipe de saúde para a atenção às doenças crônicas como HAS, a ausência de cadastramento na Estratégia Saúde da Família não influenciou o uso de medicamento contínuo entre os indivíduos que referiram diagnóstico de hipertensão no presente estudo. Vale destacar, entretanto, que o cadastramento por si só não garante o efetivo acompanhamento dos indivíduos, o que pode justificar os resultados observados.
A cessação do tabagismo e realização de atividade física, além de outras medidas como alimentação saudável, dieta hipossódica e manutenção do peso corporal são cuidados importantes e recomendados para o tratamento da hipertensão arterial 8. A associação direta entre menor uso de medicamentos e comportamentos de risco para a saúde sugere que os indivíduos que não fazem uso dos medicamentos tendem a ser menos aderentes também ao tratamento não medicamentoso. Menor utilização de anti-hipertensivos entre fumantes e entre idosos que não praticam atividade física regular foi relatada, por exemplo, por Campbell et al. 23 em estudo realizado no Canadá no ano de 2005.

Outros fatores não abordados neste trabalho são relevantes para compreender a não utilização de medicamentos de uso contínuo entre os indivíduos com hipertensão. Dentre eles podese citar: o fato de a hipertensão arterial ser uma doença assintomática que pode induzir o indivíduo a não acreditar na doença e a não utilizar os medicamentos 39 , o esquecimento das doses principalmente entre os idosos 40 , a necessidade de utilização de mais de um medicamento para obter o controle da HAS (polifarmácia) e a ocorrência de efeitos adversos como tosse seca, edema periférico e disfunção sexual, como já citado, que podem levar o indivíduo a descontinuar o uso do medicamento 31 .

A falta de especificidade da pergunta sobre uso de medicamento contínuo é uma importante limitação deste estudo. $\mathrm{O}$ ajuste para a presença de outras doenças crônicas que também exigem uso contínuo de medicamentos corrige, em parte, essa limitação. Ao realizar análise com os indivíduos que relataram apenas HAS, não se percebeu mudança na direção das associações apresentadas neste estudo, apenas a escolaridade deixou de apresentar associação estatisticamente significante (dados não apresentados). A utilização de respondente secundário consiste em um dos grandes desafios metodológicos para a análise da PNAD. Neste estudo, 29\% das respostas foram obtidas do por meio do informante secundário, mas a análise realizada apenas com aqueles que responderam ao inquérito não apresentou diferença na direção das associações observadas. Jardim et al. ${ }^{41}$ encontraram uma boa concordância entre a hipertensão arterial autorreferida e referida por informante secundário, indicando boa confiabilidade. É possível que algumas pessoas que declararam não utilizar medicamentos de uso contínuo estejam obtendo o controle da pressão arterial apenas com o tratamento não farmacológico conforme orientação das Diretrizes Brasileiras de Hipertensão. Há de se ressaltar que essa conduta é 
limitada a um curto período de tempo e para alguns indivíduos 42 .

Mesmo com a garantia de direito universal ao sistema de saúde e com as melhorias observadas após a implantação do SUS 43,44, foram verificadas desigualdades regionais, sociais e de gênero na utilização de medicamentos de uso contínuo. O controle de HAS e outras ações próprias da atenção primária são fundamentais para o enfrentamento das doenças crônicas não transmissíveis. Nossos resultados apontam al- guns desafios a serem superados e que remetem à necessidade do contínuo investimento na atenção primária, nas ações de promoção da saúde e busca da superação das iniquidades em saúde. Medidas governamentais para o acesso aos medicamentos como a implantação do Programa Farmácia Popular do Brasil podem ser benéficas, desde que o usuário não perca seu vínculo com os profissionais de saúde e seja orientado e responsabilizado pelos cuidados necessários ao controle da HAS.

\section{Resumen}

El objetivo de este estudio fue estimar la prevalencia y los factores asociados a la falta de consumo de algunos medicamentos prescritos de forma continua en las personas de 30 a 79 años que informaron hipertensión en la Encuesta Nacional por Muestra de Domicilio (PNAD-2008). Las razones de prevalencia y su IC95\% se obtuvieron mediante regresión de Poisson. La proporción de personas que no consumen medicamentos de forma continuada fue de un 17\%. Características positivamente asociadas con la falta de consumo fueron: sexo masculino, residencia en el Norte, Noreste, Centro Oeste y el tabaquismo. El hecho de no consumir estos medicamentos disminuye con la edad, los ingresos per cápita del hogar, número de enfermedades crónicas y la asistencia a una consulta médica en los últimos 12 meses; fue menor entre los que tenían 11 o más años de escolaridad, que entre quienes no trabajaban o estaban desempleados y no participaban en actividades físicas. La adhesión a la terapia de la medicación en el control de la hipertensión es necesaria y requiere una inversión en la atención primaria, mejorar el acceso a servicios de salud y hacer frente a las desigualdades regionales, sociales y de género.

Hipertensión; Medicamentos de Uso Contínuo; Terapéutica

\section{Colaboradores}

R. A. Ferreira, S. M. Barreto e L. Giatti trabalharam na concepção, delineamento, análise e redação do artigo. S. M. Barreto trabalhou na concepção, delineamento e redação do artigo.

\section{Agradecimentos}

L. Giatti foi bolsista do Programa de Apoio a Projetos Institucionais com a Participação de Recém-Doutores (PRODOC), da Capes, durante a realização deste manuscrito. S. M. Barreto é bolsista de produtividade em pesquisa do CNPq (processo no 300159/99-4). 


\section{Referências}

1. World Health Organization. Non-communicable diseases country profile, 2011. http://www.who. $\mathrm{int} / \mathrm{nmh} /$ publications/ncd_profiles2011/en/in dex.html (acessado em 24/Set/2012).

2. Lim SS, Vos T, Flaxman AD, Danaei G, Shibuya K, Adair-Rohani $\mathrm{H}$, et al. A comparative risk assessment of burden of disease and injury attributable to 67 risk factors and risk factor clusters in 21 regions, 1990-2010: a systematic analysis for the Global Burden of Disease Study 2010. Lancet 2012; 380:2224-60.

3. Kearney PM, Whelton M, Reynolds K, Muntner P, Whelton PK, He J. Global burden of hypertension: analysis of worldwide data. Lancet 2005; 365: 217-23.

4. Moreira JPL, Moraes JR, Raggio RL. Prevalence of self-reported systematic arterial hypertension in urban and rural enviroments in Brazil: a population-based study. Cad Saúde Pública 2013; 29: 62-72.

5. Secretaria de Vigilância em Saúde/Secretaria de Gestão Estratégica e Participativa, Ministério da Saúde. Vigitel Brasil 2011: vigilância de fatores de risco e proteção para doenças crônicas por inquérito telefônico. Brasília: Ministério da Saúde; 2012.

6. Chobanian AV, Bakris GL, Black HR, Cushman WC, Green LA, Izzo JL, et al. The seventh report of the Joint National Committee on Prevention, Detection, Evaluation, and Treatment of High Blood Pressure: the JNC 7 report. JAMA 2003; 289: 2560-72.

7. McAlister FA, Wilkins $\mathrm{K}$, Joffres $\mathrm{M}$, Leenen $\mathrm{FH}$ Fodor G, Gee M, et al. Changes in the rates of awareness, treatment and control of hypertension in Canada over the past two decades. CMAJ 2011; 183:1007-101.

8. Centers of Disease Control and Prevention. Vital signs: prevalence, treatment and control of hypertension. United States, 1999-2002 and 2005-2008. MMWR Morb Mortal Wkly Rep 2011; 60:103-8.

9. Kearney PM, Whelton M, Reynolds K, Whelton PK, He J. Worldwide prevalence of hypertension: a systematic review. J Hypertens 2004; 22:11-9.

10. Gus I, Harzheim E, Zaslavsky C, Medina C, Gus M. Prevalence, awareness and control of systemic arterial hypertension in the state of Rio Grande do Sul. Arq Bras Cardiol 2004; 83:429-33.

11. Pereira MR, Coutinho MSSA, Freitas PF, D'Orsi E, Bernardi A, Hass R. Prevalência, conhecimento, tratamento e controle da hipertensão arterial sistêmica na população adulta urbana de Tubarão, Santa Catarina, Brasil, em 2003. Cad Saúde Pública 2007; 23:2363-74.

12. Firmo JOA, Barreto SM, Lima-Costa MF. The Bambui Health and Aging Study (BHAS): factors associated with the treatment of hypertension in older adults in the community. Cad Saúde Pública 2003; 19:817-27.
13. Damasceno A, Azevedo A, Silva-Matos C, Prista A, Diogo B, Lunet N. Hypertension, prevalence, awareness, treatment and control in Mozambique. Hypertension 2009; 54:77-83.

14. Mendéz-Chacón E, Santamaria-Ulloa C, RoseroBixby L. Factors associated with hypertension prevalence, unawareness and treatment among Costa Rica elderly. BMC Public Health 2008; 8:275.

15. Instituto Brasileiro de Geografia e Estatística. Pesquisa Nacional por Amostra de Domicílios 2008: notas metodológicas pesquisa básica, pesquisa especial de tabagismo e pesquisas suplementares de saúde e acesso à internet e posse de telefone móvel celular para uso pessoal. Rio de Janeiro: Instituto Brasileiro de Geografia e Estatística; 2009.

16. Lima-Costa MF, Peixoto SV, Firmo JO. Validade da hipertensão arterial autorreferida e seus determinantes (projeto Bambuí). Rev Saúde Pública 2004; 38:637-42.

17. Chrestani MA, Santos IS, Matiasevich AM. Hipertensão arterial sistêmica autorreferida: validação diagnóstica em estudo de base populacional. Cad Saúde Pública 2009; 25:2395-406.

18. Barros MB, Francisco PM, Zanchetta LM, César CL. Tendências das desigualdades sociais e demográficas na prevalência de doenças crônicas no Brasil, PNAD: 2003-2008. Ciênc Saúde Coletiva 2011; 16:3755-68

19. Silva PL, Pessoa DG, Lila MF. Análise estatística de dados da PNAD: incorporando a estrutura do plano amostral. Ciênc Saúde Coletiva 2002; 7:659-70.

20. Gontijo MF, Ribeiro AQ, Klein CH, Rozenfeld S, Acúrcio FA. Uso de anti-hipertensivos e antidiabéticos por idosos: inquérito de Belo Horizonte, Minas Gerais, Brasil. Cad Saúde Pública 2012; 28:1337-46

21. Osório RG, Servo LM, Piola SF. Necessidade de saúde insatisfeita no Brasil: uma investigação sobre a não procura de atendimento. Ciênc Saúde Coletiva 2011; 16:3741-53.

22. Travassos C, Oliveira EX, Viacava F. Desigualdades geográficas e sociais no acesso aos serviços de saúde no Brasil: 1998 e 2003. Ciênc Saúde Coletiva 2006; 11:975-86.

23. Campbell NRC, So L, Amankwah E, Quan H, Maxwell C. Characteristics of hypertensive Canadians not receiving drugs therapy. Can J Cardiol 2008; 24:485-90.

24. Wu Y, Huxley R, Li L, Anna V, Xie G, Yao C, et al. Prevalence, awareness, treatment and control of hypertension in China: data from China National Nutrition and Health Survey 2002. Circulation 2008; 118:2679-86.

25. Szwarcwald CL, Souza-Júnior PR, Damacena GN. Socioeconomic inequalities in the use of outpatient services in Brazil according to helth care need: evidence from the World Health Survey. BMC Health Serv Res 2010; 10:217. 
26. Travassos C, Viacava F, Pinheiro R, Brito A. Utilização dos serviços de saúde no Brasil: gênero, características familiares e condição social. Rev Panam Salud Pública 2002; 11:365-73.

27. Bertoldi AD, Barros AJD, Hallal PC, Lima RC. Utilização de medicamentos em adultos: prevalência e determinantes individuais. Rev Saúde Pública 2004 ; 38:228-38.

28. Moreira JP, Moraes JR, Luiz RR. Utilização de consulta médica e hipertensão arterial sistêmica nas áreas urbanas e rurais do Brasil, segundo dados da PNAD 2008. Ciênc Saúde Coletiva 2011; 16 : 3781-93.

29. Pinheiro RS, Viacava R, Travassos C, Brito AS. Gênero, morbidade, acesso e utilização de serviços de saúde no Brasil. Ciênc Saúde Coletiva 2002; 7:687-707.

30. Nations M, Firmo JOA, Lima-Costa MF, Uchôa E. Balking blood pressure "control" by older persons of Bambuí, Minas Gerais State, Brazil: an ethnoepidemiological inquiry. Cad Saúde Pública 2011; 27 Suppl 3:S378-89.

31. Munger MA, Tassell BW, LaFleur J. Medication nonadherence: an unrecognized cardiovascular risk factor. MedGenMed 2007; 9:58.

32. Lee HS, Park YM, Kwon HS, Lee JH, Park YJ, Lim SY, et al. Prevalence, awareness, treatment, and control of hypertension among people over 40 years old in a rural area of South Korea: The Chungju Metabolic Disease Cohort (CMC) Study. Clin Exp Hypertens 2010; 32:166-78.

33. Barreto SM, Figueiredo RC. Doença crônica, autoavaliação em saúde e comportamento de risco: diferença de gênero. Rev Saúde Pública 2009; 43 Suppl 2:S38-47.

34. Galobardes B, Shaw M, Lawlor DA, Lynch JW, Smith GD. Indicators of socioeconomic position (part 1). J Epidemiol Community Health 2006; 60:7-12.
35. Schmidt MI, Duncan BB, Azevedo e Silva G, Menezes AM, Monteiro CA, Barreto SM, et al. Chronic non-communicable diseases in Brazil: burden and current challenges. Lancet 2011; 377:1949-61.

36 Viacava F. Acesso e uso de serviços de saúde pelos brasileiros. Radis 2010; 96:12-9.

37. Ministério da Saúde. Portaria no 184, de 3 de fevereiro de 2011. Dispõe sobre o Programa Farmácia Popular do Brasil. Diário Oficial da União 2011; 4 fev.

38. Giatti L, Barreto SM. Situação no mercado de trabalho e utilização dos serviços de saúde no Brasil. Ciênc Saúde Coletiva 2011; 16:3817-27.

39. Firmo JOA, Peixoto SV, Loyola Filho AI, Uchôa E, Lima-Costa MF. Birth cohort differences in hypertension control in a Brazilian population of older elderly: the Bambuí cohort study of aging (1997 and 2008). Cad Saúde Pública 2011; 27 Suppl 3:S427-34.

40. Paniz VMV, Fassa AG, Facchini LA, Piccini RX, Tomasi E, Thumé E, et al. Free access to hypertension and diabetes medicines among the elderly: a reality yet to be constructed. Cad Saúde Pública 2010; 26:1163-74.

41. Jardim R, Barreto SM, Giatti L. Confiabilidade das informações obtidas de informante secundário em inquéritos de saúde. Cad Saúde Pública 2010; 26:1537-48.

42. Sociedade Brasileira de Cardiologia; Sociedade Brasileira de Hipertensão; Sociedade Brasileira de Nefrologia. VI diretrizes brasileiras de hipertensão. Arq Bras Cardiol 2010; 95(1 Suppl 1): 1-51.

43. Macinko J, Lima-Costa MF. Horizontal equity in health care utilization in Brazil, 1998-2008. Int J Equity Health 2012; 11:33.

44. Paim J, Travassos C, Almeida C, Bahia L, Macinko J. The Brazilian health system: history, advances, and challenges. Lancet 2011; 377:1778-97.

Recebido em 25/Nov/2012

Versão final reapresentada em 05/Set/2013 Aprovado em 10/Out/2013 